\title{
Modeling and Simulation Tool for Sustainable MC Supply Chain Design and Assessment
}

\author{
Paolo Pedrazzoli, Marino Alge, Andrea Bettoni, and Luca Canetta \\ University of Applied Sciences and Arts of Southern Switzerland, ICIMSI, Manno, Switzerland \\ \{paolo.pedrazzoli, marino.alge, andrea.bettoni, \\ luca.canetta\} @supsi.ch
}

\begin{abstract}
Supply chain design, management and assessment are key success drivers in nowadays globalised economy. With the advent of new paradigms such as sustainability and mass customization, a new generation of tools is required. This work presents a supply chain simulation tool that allows to take into account the specificity of mass customized markets, efficiently dealing with the adjustable product physical structure and the complexity of handling customized Lot Size One orders. Moreover, this tool is integrated with a sustainable Assessment Engine that allows to configure, since the product design phase, the entire supply chain in a lifecycle perspective. In order to ensure a wide applicability of the tool, this is developed using a client-server architecture and exploiting a Shared Data Model that facilitates the integration of many applications coming from different providers thus giving a powerful decision support tool to companies' decision makers.
\end{abstract}

Keywords: supply chain, simulation tools, mass customization, sustainability.

\section{Introduction}

Mass customization is nowadays an established paradigm that companies, belonging to many different sectors, apply in order to differentiate their proposal and to allow a better fitting of their products with customers' needs. However its implementation is not yet a simple and straightforward process as it deeply depends on the specific characteristics of the reality where it is put into practice. This asks for methodologies to be used as guidelines and specific tools that support the integrated product-processsupply chain design brought forth by mass customization. Unfortunately such comprehensive approaches are, to these days, still lacking [1].

At the same time, the mere evaluation of economic performances is no more suitable in the current global economy. As the concept of sustainability is getting more and more consideration along with customers growing awareness about it, social and environmental issues related to the company business need to be taken into account towards greener and fairer products and supply chains. In the next future, such topics will increasingly set the governments' agenda and drive their decisions - some are already in place - in a shape that could considerably impact on the companies' business. However companies still struggle in finding adequate assessment frameworks for measuring sustainability performance of their supply chains [2]. 
To this end, within the S-MC-S project, that is co-funded by the European Commission, a collaborative software environment, we will refer to as the S-MC-S System, has been created. This system enables a collaborative and holistic design of a mass customized stable solution space [3] providing a twofold benefit: on the one hand, it allows different design tools covering different compartments of the whole project to share data thus providing support in the handling of the huge and heterogeneous information related to a mass customized solution space; on the other hand, it allows the gathering and formalization of the data needed to carry out a sustainability assessment of the designed solution all along its lifecycle.

This paper will present the conceived environment starting from an overview of the software architecture and Shared Data Model of the proposed system. In the second part, the development of a specific tool for the modeling and simulation of a supply chain for mass customized production will be described. Such tool is meant to overcome the weaknesses of the traditional approaches and to deliver a tool capable to cope with the complex logics of Lot Size One production and delivery driven by mass customization.

\section{Shared Data Model}

As previously said, the first step for laying the foundations of an integrated environment has been the definition of a Shared Data Model [3] where all the involved tools can store and retrieve the relevant information concerning the solution space. This description covers the three main aspects of a product in a lifecycle perspective. First of all the product nature, both in its hierarchical structure and its physical properties. Then the production process which encompasses the whole life of a product from its raw materials extraction down to its end of life treatment. Finally the supply chain, which describes all the involved actors and the carried out transportations of resources which have a role in the product life.

This collaborative description of the product is then used to perform a detailed assessment of its sustainability level. The assessment performed at the design stage of a product enables an iterative feedback between the design choices and the assessment-resulting KPIs based on a sustainable strategy of the company. Detection of problems and investigation of alternatives at this stage is crucial since late recognition and handling of these issues prevents from any effective countermeasures implementation.

In order to enable design of the solution space, a data model has been developed. This data model becomes the common language platform on which all data-providing agents exchange information with the underlining system, thus allowing interaction between them and ensuring consistency of correlated data. Inside the data model five macro areas can be identified as shown in the high-level class diagram of Fig. 1:

- core data entities: contains the project root node and provides elementary building blocks like resources and facilities along with their possible specialized entities as well as descriptive bridges for the other areas; 
- product area: models both the resources that compose the product physical structure and how customization choices change the physical structure of the product;

- process area: models the operations which are connected by flows of materials as well as their input and transformed output;

- supply chain area: models the facilities involved in the solution space and the transportations in between them;

- assessment properties area: models assessment properties, representing the characteristics of the data entities used by the Assessment Engine for its calculations.

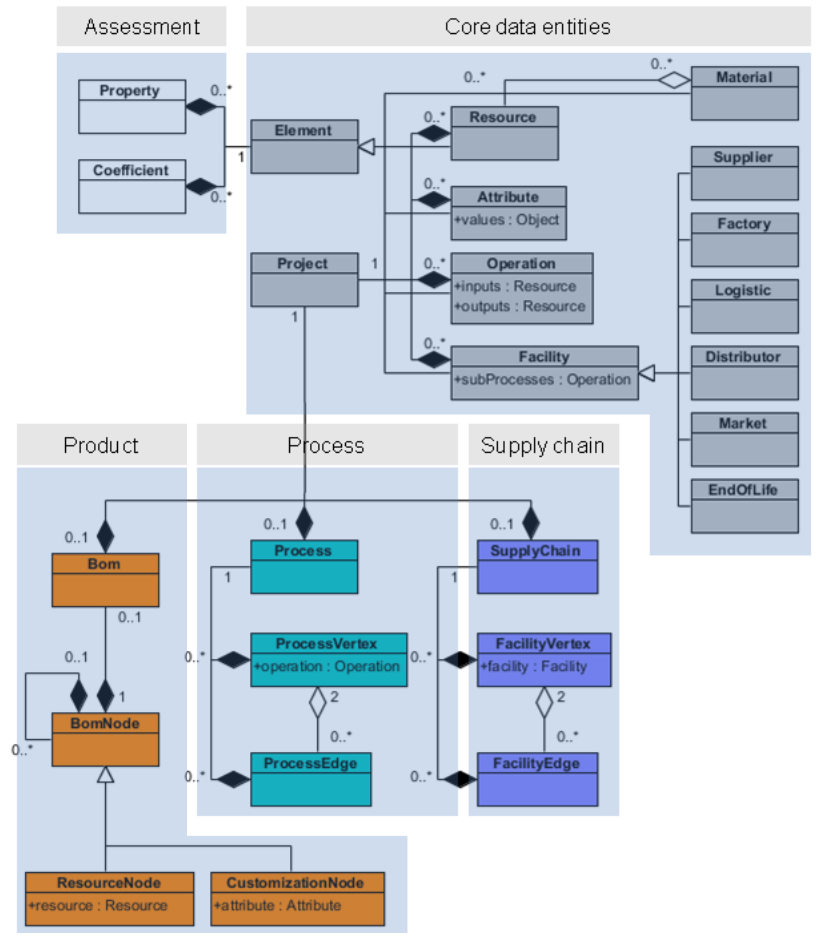

Fig. 1. UML Class Diagram of the Shared Data Model

\section{System Architecture}

The second building block of the S-MC-S System is its conceived architecture that is meant to structure the interactions amongst the various design and assessment tools relying on the above described data model. This architecture is based on a clientserver model where the server, embodied by the Assessment Engine in Fig. 2, offers specific services and functionalities aimed to implement the full potential of the Shared Data Model platform and of a centralized assessment computation. The server, first of all, handles the persistency of all solution space data, lifting the burden of the developing effort of client specific persistency and providing a centralized repository of shared data on which clients can work collaboratively. The server also exposes 
locking mechanisms upon shared data to avoid data concurrent modification and to ensure the overall consistency. Moreover the server does not only provide services to handle shared data but it can be also triggered to perform the assessment computation based on the selected assessment model and to return the detailed assessment results back to the calling design tool.

By proposing this architecture the effort to develop a client able to take advantage of the S-MC-S services or even to adapt an existing design tool to it is minimized thus fostering the adoption of the S-MC-S System.

This is the case of the presented supply chain design tool, whose detailed architecture is shown in Fig. 2, where the server part is just sketched for completeness.

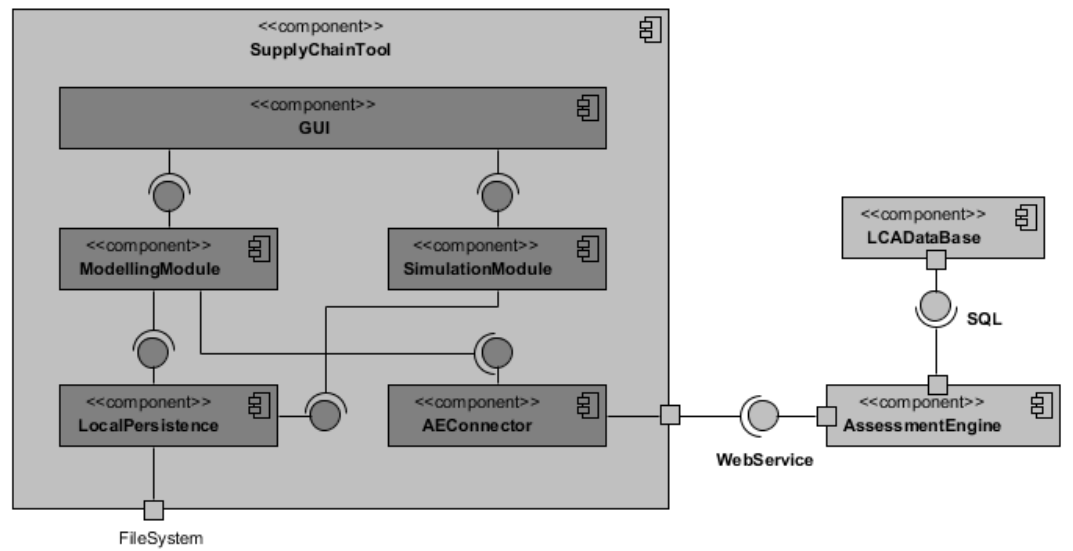

Fig. 2. UML Component Diagram of the Supply Chain Tool

\section{Supply Chain Modeling Tool}

Within the context of sustainable production of mass customized goods, as it has been aforementioned, the need for better coordination of the actors involved in the supply chain is recognized as one of the key success factors towards the paradigm adoption. Selection of proper suppliers becomes essential in order to handle the required flexibility needed to sustain the several choices offered to the final customer in an order-driven production. At the same time the ability to predict the system capability to satisfy the incoming orders within the allowed lead time is mandatory if an efficient supply chain is to be set up.

However, the existing supply chain modeling tools don't cope with such a level of detail. Here the customer order is no more an abstract concept representing a product lot to be delivered in time, being aggregated with other customer orders and managed in an undifferentiated way. It has to be represented as a single entity requiring a specific customization of the offered good. To this purpose a tool meant to support modeling and simulation of a mass customization oriented supply chain is here presented. 
The definition of the supply chain attains two different levels, logical and geographical supported by related editing panels shown in Fig. 3. The first one allows to model into a graph structure even complex supply chains by defining actors, the macro-processes they carry out and the input/output resources for each one. The second one allows to intuitively geo-position the facilities and to retrieve distances and routes between them for sustainability impact calculation.

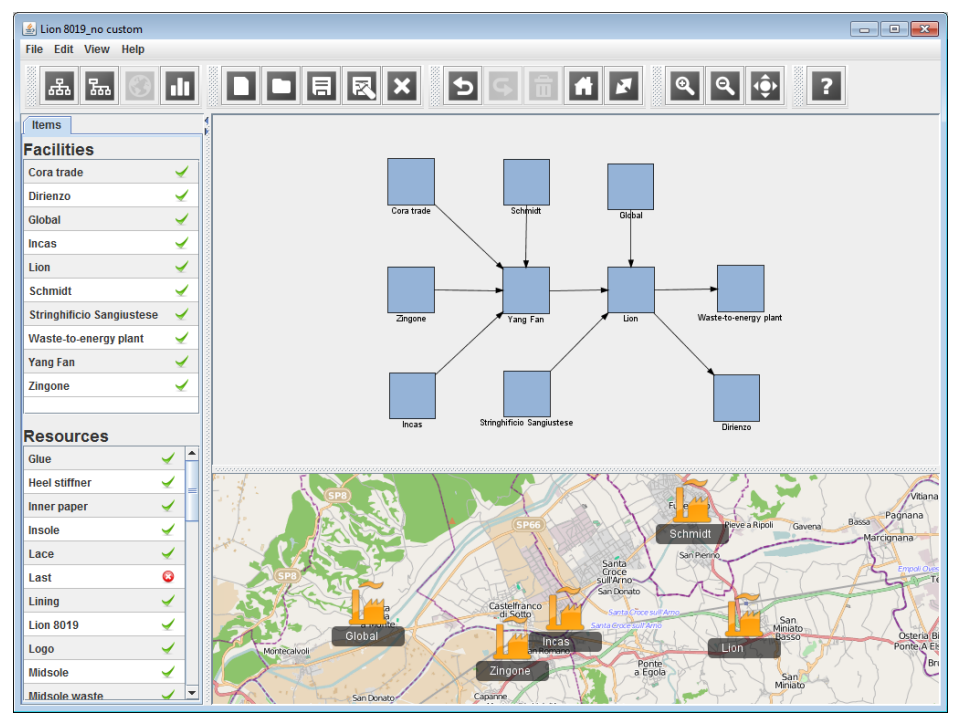

Fig. 3. Screenshot of the supply chain modeling interface

\section{Simulation of the Supply Chain Behavior}

The simulation feature of the supply chain tool acts as a decision support platform that enable, at low cost and robust simulation environment, analysis of what-if situations in order to compare alternative supply chains [5]. Furthermore it allows to detect problematic situations like bottlenecks, starving conditions, bullwhip effects under various simulation parameterizations.

This feature exploits a Discrete Event Simulation engine that, upon description of the stochastically variable events occurring in the supply chain, computes its behavior. The tool is provided with an animation feature that allows to graphically verify that the modeled solution is properly working.

One innovative aspect of this supply chain simulation tool is the enhanced description of the product customization aspect. This means that the product samples generated as demand of the various simulated markets are indeed product instances configured from the solution space according to the user-defined product mix. These configurations vary the nature of the different macro-processes applied on each single configured product resulting in different input resources requirements and different output resources productions, as well as different capacity loads and consequent lead times. 
For each simulated facility, different strategies and methods can be defined and resulting behaviors simulated. At factory level, production capabilities are triggered by events generated either following a pull or push strategy thus embodying plan of make-to-order, assembly-to-order or make-to-stock productions. At suppliers level, supply agreements which drive the supplier behavior can be postulated for each of the replenished items. Moreover, at markets level, orders generation is driven by its parameterized statistical distribution aimed at representing the expected product mix. The features described above allow to model and simulate typical mass-customization supply chains supporting definition of different decoupling points for the handled resources. This enables proper representation of the different strategies used in order to achieve mass customized goods where standard parts are usually provided make-tostock whereas highly customized components are produced or supplied only upon reception of customers orders.

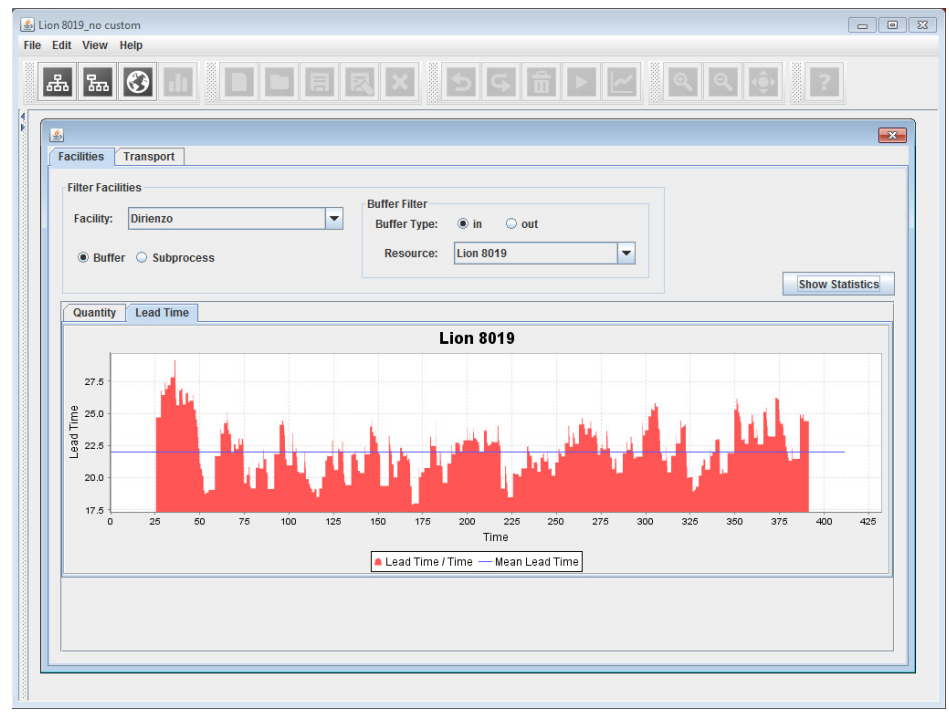

Fig. 4. Screenshot of a sample simulation result (order lead time)

As shown in the example of Fig. 4, the simulation results are summarized into an overall supply chain score though they can also be browsed for each facility and for each stock and process linked to it. In this way, it is easier to identify critical processes or facilities and study them deeper into details. Information like order lead time, stock level and stock related costs are available for each resource and the capacity utilization of each production line can also be analyzed.

\section{Sustainability Assessment of the Supply Chain}

Another evaluation layer proposed as support for suppliers selection is the sustainability one. Being connected to the multi-tool S-MC-S System environment, 
the presented supply chain design tool can exploit the holistic definition of the solution space held in the Shared Data Model in order to calculate its sustainability performances.

Indeed the tool itself completes the information necessary for carrying out the assessment that lie at supply chain level as, for instance, the distances of the covered transportations and the used means or the energy mix used by the facilities based on their geo-position. On the other hand, reuse of detailed definition of the product and production process, defined in other tools, allows to rapidly perform a reliable and complete assessment of the lifecycle sustainability impact of the product solution space.

The outcome [6] consists in the estimation of a total of 40 sustainability indicators (17 environmental, 10 economic and 13 social).

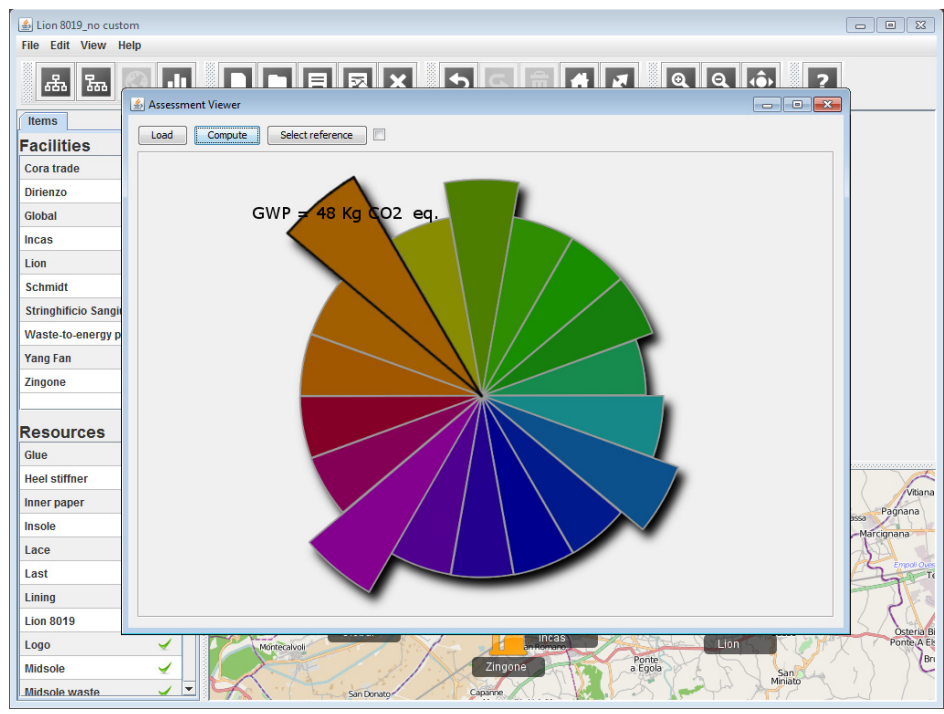

Fig. 5. Screenshot of the displaying of the sustainability assessment results

\section{Conclusions}

European Industry is developing methods and enabling technologies towards a customer-oriented and sustainable manufacturing as a key answer to global competitive pressure. This trend has been also understood by policy maker at the European Commission (e.g. as per the "Factory of the Future" multi-annual road-map [7]), and embraced by funding programmes for industrial research (FP7 and Horizon $2020[8])$.

The challenges related with customization lay with the need to efficiently deal with adjustable product physical configuration (whose options must be clearly identified and limited), with the complexity of producing and handling customized Lot Size One orders, and with the need to cope with sustainability requirements. 
The research work here presented focuses on the development of a decision support tool meant to cope with the definition of a proper supply chain for sustainable mass customized products, where current tools fall short. This is done in the framework of the S-MC-S project, that promotes the development of a software environment for the collaborative design of a sustainable mass customized solution space that takes into account product, process and supply chain.

The tool developed uses a client-server architecture and exploits a Shared Data Model that facilitates the integration of many different design tools coming from several providers. The tool empowers a better coordination of the actors involved in the supply chain recognized in the context of sustainable production of mass customized goods, which was one of the limiting factors of mass customization successful implementation. Indeed it supports, through an adherent to reality simulation, the selection of proper suppliers capable to handle the required flexibility in an order-driven production and, at the same time, it fosters the ability to predict the system capability to satisfy the incoming orders within the allowed lead time.

\section{References}

1. Castellano, E., Dolado, J.: Product-processes-supply chain structures alignment for mass customization scenarios. A literature review. In: 4th International Conference on Industrial Engineering and Industrial Management. San Sebastián (2010)

2. Hassini, E., Surti, C., Searcy, C.: A literature review and a case study of sustainable supply chains with a focus on metrics. I. J. of Production Economics 140, 69-82 (2012)

3. Piller, F.T.: Mass Customization: Reflections on the State of the Concept. I. J. of Flexible Manufacturing Systems 16(4), 313-334 (2004)

4. Bettoni, A., Alge, M., Rovere, D., Pedrazzoli, P., Canetta, L.: Towards Sustainability Assessment: Reference Data Model for Integrated Product, Process, Supply Chain Design. In: Proceedings of the 18th International ICE Conference on Engineering, Technology and Innovation. IEEE Press, New York (2012)

5. Chang, Y., Makatsoris, H.: Supply Chain Modeling Using Simulation. I. J. of Simulation 2(1), 24-30 (2000)

6. Canetta, L., Pedrazzoli, P., Sorlini, M., Bettoni, A., Boër, C.R., Corti, D.: Customization and Manufacturing Sustainability: General considerations and footwear investigation. In: Piller, F.T., Chesbrough, H. (eds.) Bridging Mass Customization \& Open Innovation. Lulu, Raleigh (2011)

7. European Commission: Factories of the Future PPP Strategic Multi-Annual Roadmap. Publications Office of the European Union, Luxembourg (2012)

8. Community Research and Development Information Service,

http: //cordis. europa.eu 